\title{
Properties of Commuting Graphs over Semidihedral Groups
}

\author{
Tao Cheng ${ }^{1}$, Matthias Dehmer ${ }^{2,3,4,5, *}$, , Frank Emmert-Streib ${ }^{6,7}$, Yongtao Li ${ }^{8}$ and Weijun Liu ${ }^{9}$ \\ 1 School of Mathematics and Statistics, Shandong Normal University, Jinan 250358, China; \\ taocheng@sdnu.edu.cn \\ 2 Department of Computer Science, Swiss Distance University of Applied Sciences, 3900 Brig, Switzerland \\ 3 College of Artificial Intelligence, Nankai University, Tianjin 300350, China \\ 4 School of Sciences, Xi'an Technological University, Xi'an 710021, China \\ 5 Department of Biomedical Computer Science and Mechatronics, UMIT, A-6060 Hall in Tyrol, Austria \\ 6 Predictive Medicine and Data Analytics Lab, Department of Signal Processing, Tampere University, \\ 33100 Tampere, Finland; frank.emmert-streib@tuni.fi \\ 7 Institute of Biosciences and Medical Technology, 33520 Tampere, Finland \\ 8 School of Mathematics, Hunan University, Changsha 410082, China; ytli0921@hnu.edu.cn \\ 9 School of Mathematics and Statistics, New Campus, Central South University, Changsha 410083, China; \\ wjliu@csu.edu.cn \\ * Correspondence: matthias.dehmer@umit.at
}

check for

updates

Citation: Cheng, T.; Dehmer, M.;

Emmert-Streib, F.; Li, Y.; Liu, W.

Properties of Commuting Graphs over Semidihedral Groups. Symmetry 2021, 13, 103. https://doi.org/ $10.3390 /$ sym 13010103

Received: 7 December 2020 Accepted: 6 January 2021 Published: 8 January 2021

Publisher's Note: MDPI stays neutral with regard to jurisdictional clai$\mathrm{ms}$ in published maps and institutional affiliations.

\section{Copyright: (๑) 2021 by the authors. Li-} censee MDPI, Basel, Switzerland. This article is an open access article distributed under the terms and conditions of the Creative Commons Attribution (CC BY) license (https:// creativecommons.org/licenses/by/ $4.0 /)$.

\begin{abstract}
This paper considers commuting graphs over the semidihedral group $S D_{8 n}$. We compute their eigenvalues and obtain that these commuting graphs are not hyperenergetic for odd $n \geq 15$ or even $n \geq 2$. We further compute the Laplacian spectrum, the Laplacian energy and the number of spanning trees of the commuting graphs over $S D_{8 n}$. We also discuss vertex connectivity, planarity, and minimum disconnecting sets of these graphs and prove that these commuting graphs are not Hamiltonian.
\end{abstract}

Keywords: graph spectrum; semidihedral groups; commuting graph; spectral radius

\section{Introduction}

The study of spectrum of commuting graphs of a given group or a ring attracted a large amount of attention in recent years. The concept of commuting graph has been defined in [1]. Let $G$ be a group and $X$ be a nonempty subset of $G$. The commuting graph $\mathcal{C}(G, X)$ has the vertex set $X$ where $x_{1}, x_{2} \in X$ are adjacent whenever $x_{1} x_{2}=x_{2} x_{1}$ in $G$. In the case that $X=G$, we denote $\Gamma(G)=\mathcal{C}(G, G)$ for short. Various aspects of commuting graphs of finite groups can be found in [2] and its references.

In this paper, we only consider finite simple graphs on vertex set $\left\{v_{1}, v_{2}, \ldots, v_{n}\right\}$, i.e., graphs without multiple-edges and loops on a finite vertex set. We use the following standard notation; see, e.g. [3,4]. We associate $\Gamma$ with a symmetric real $n \times n$ matrix $A(\Gamma)=\left(a_{i j}\right)_{i, j=1}^{n}$, called adjacency matrix, where $a_{i j}=1$ if the vertex $v_{i}$ is adjacent to $v_{j}$, and 0 otherwise. The characteristic polynomial of $\Gamma$ is $p(\lambda)=\operatorname{det}(\lambda I-A(\Gamma))$. These eigenvalues with their multiplicity compose the spectrum of graph $\Gamma$. Recall that the spectral radius of a matrix $A$, denoted by $\rho(A)$, is defined by $\rho(A)=\max \left\{\left|\lambda_{i}(A)\right|: i=1,2, \ldots, n\right\}$.

Let $D(\Gamma)$ denote the diagonal matrix of degrees of a graph $\Gamma$. The Laplacian matrix of $\Gamma$ is the matrix $L(\Gamma)=D(\Gamma)-A(\Gamma)$, and the Laplacian spectrum of $\Gamma$ is a multiset given by L-spec $(\Gamma)=\left\{\mu_{1}^{m_{1}}, \mu_{2}^{m_{2}}, \ldots, \mu_{l}^{m_{l}}\right\}$, where $\mu_{1}, \mu_{2}, \ldots, \mu_{l}$ are the Laplacian eigenvalues of $\Gamma$ with multiplicities $m_{1}, m_{2}, \ldots, m_{l}$.

The energy of the graph $\Gamma$ is defined as the sum of the absolute values of the eigenvalues of its adjacency matrix, that is, $E(\Gamma)=\sum_{k=1}^{n}\left|\lambda_{k}(\Gamma)\right|$. This concept was introduced in 1978 by Gutman [5]. Clearly, the energy of complete graph $K_{n}$ of order $n$ is $2(n-1)$. A graph $\Gamma$ is said to be hyperenergetic if $E(\Gamma)>E\left(K_{n}\right)=2(n-1)$ and nonhyperenergetic if $E(\Gamma) \leq 2(n-1)$. It is known [6](Theorem 5.24) that for almost all graphs $\Gamma$, 
$E(\Gamma)>(1 / 4+o(1)) n^{3 / 2}$, which means that almost all graphs are hyperenergetic. Therefore, the problem of finding nonhyperenergetic graphs has been extremely meaningful, see [7]. Additionally, the Laplacian energy, denoted by $L E(\Gamma)$, is defined as $L E(\Gamma)=$ $\sum_{i=1}^{l}\left|\mu_{i}-\frac{2 m}{n}\right|$, where $m$ is the number of edges in the graph $\Gamma$. Both the energy and the Laplacian energy of graphs play an important role in solving many physical and chemical problems. In theoretical chemistry, the energy of a given molecular (conjugated hydrocarbons) graph approximately describes the total $\pi$-electron energy of the molecule represented by that graph, see the comprehensive survey $[6,8]$ for more details.

Let $\Gamma$ be a simple graph and $u$ be a vertex. The neighbor set of $u$ in $\Gamma$, denoted by $\mathbb{N}(u)$, is the set of all vertices adjacent to $u$. The degree of $u$ is $d(u)=|\mathbb{N}(u)|$. The minimum degree of $\Gamma$ is denoted by $\sigma(\Gamma)$. A spanning tree of $\Gamma$ is a connected subgraph of $\Gamma$ on all vertices with $|V(\Gamma)|-1$ edges, which is in fact a tree on $V(\Gamma)$. The number of spanning trees in a graph $\Gamma$ is denoted by $\operatorname{st}(\Gamma)$.

A simple graph is said to be planar if it can be drawn on the plane with no crossing edges. The vertex-connectivity of $\Gamma$ is denoted by $\kappa(\Gamma)$, which is the minimum number of vertices whose deletion will result in a disconnected graph or trivial graph. A set $S \subset E(\Gamma)$ is called a disconnecting set if its deletion will increase the number of components of $\Gamma$, and $S$ is called a minimal disconnecting set if there is no proper subset of $S$ that disconnects $\Gamma$. For two vertex sets $U$ and $V$, we denote $\mathbb{E}[U, V]$ by the set of edges having one end-point in $U$ and the other in $V$.

Our focus in this paper lies on the semidihedral group

$$
S D_{8 n}=\left\langle a, b \mid a^{4 n}=b^{2}=e, b a b=a^{2 n-1}\right\rangle
$$

for $n \geq 2$. Moreover, all the $8 n$ elements of $S D_{8 n}$ could be given as

$$
S D_{8 n}=\left\{e, a, a^{2}, \ldots, a^{4 n-1}, b, b a, b a^{2}, \ldots, b a^{4 n-1}\right\} .
$$

From Lemma 10 of [9], we have $\Gamma\left(S D_{8 n}\right)=K_{4} \vee\left(n K_{4} \cup K_{4 n-4}\right)$ for odd $n$, and $\Gamma\left(S D_{8 n}\right)$ $=K_{2} \vee\left(2 n K_{2} \cup K_{4 n-2}\right)$ for even $n$.

Remark 1. If $n$ is odd, then $Z\left(S D_{8 n}\right)=\left\{e, a^{n}, a^{2 n}, a^{3 n}\right\}$ is the center of semidihedral group. Now, we suppose that $\Phi_{1}=\left\{e, a, a^{2}, \ldots, a^{4 n-1}\right\}, \Phi_{2}=\left\{b, b a, b a^{2}, \ldots, b a^{4 n-1}\right\}=\cup_{i=0}^{n-1} \Phi_{2}^{i}$, where $\Phi_{2}^{i}=\left\{b a^{i}, b a^{n+i}, b a^{2 n+i}, b a^{3 n+i}\right\}$ and $\Phi_{3}=\Phi_{1} \backslash Z\left(S D_{8 n}\right)$ are the subsets of $S D_{8 n}$. It is easy to check that $x y=y x$ for all $x, y \in \Phi_{2}^{i}$. However, $x y \neq y x$, when $x \in \Phi_{2}$ and $y \in \Phi_{3}$. If $n$ is even, then $Z\left(S D_{8 n}\right)=\left\{e, a^{2 n}\right\}$ is the center of semidihedral group. Similarly, we suppose that $\Psi_{1}=\left\{e, a, a^{2}, \ldots, a^{4 n-1}\right\}, \Psi_{2}=\left\{b, b a, b a^{2}, \ldots, b a^{4 n-1}\right\}=\cup_{i=0}^{n-1} \Psi_{2}^{i}$, where $\Psi_{2}^{i}=\left\{b a^{i}, b a^{2 n+i}\right\}$ and $\Psi_{3}=\Psi_{1} \backslash Z\left(S D_{8 n}\right)$ are the subsets of $S D_{8 n}$. It is also easy to find that $x y=y x$ for all $x, y \in \Psi_{2}^{i}$. However, $x y \neq y x$, if $x \in \Psi_{2}$ and $y \in \Psi_{3}$.

In the existing literature, there are still many gaps in finding characteristic polynomials, spectral radius, graph energy, Laplacian spectrum, vertex-connectivity, planarity, and Hamiltonian graphs of commuting graphs of finite semidihedral groups $S D_{8 n}$. We will address some of these problems in this paper. When $n$ has a large number of factors, it is not easy to obtain explicit expression for the eigenvalues of the graph $\Gamma\left(S D_{8 n}\right)$, so that we resort to bounding the spectral radius in such cases. Moreover, we prove that the commuting graph $\Gamma\left(S D_{8 n}\right)$ is not hyperenergetic when odd $n \geq 15$ or even $n \geq 2$. In addition, we obtain the characteristic polynomial of the Laplacian matrix of $\Gamma\left(S D_{8 n}\right)$, and we also derive the Laplacian spectrum of $\Gamma\left(S D_{8 n}\right)$. Moreover, we calculate the Laplacian energy and the number of spanning trees of $\Gamma\left(S D_{8 n}\right)$. Finally, we discuss vertex connectivity, planarity, and minimum disconnecting sets of commuting graphs of the semidihedral group $S D_{8 n}$ and prove that these commuting graphs are not Hamiltonian. 


\section{Spectral Properties of Commuting Graphs}

In the present section, we are going to find the characteristic polynomials of commuting graphs of semidihedral groups and discuss their spectral radius. Additionally, we also obtain the lower and upper bounds on the energy of the commute graph $\Gamma\left(S D_{8 n}\right)$, and prove that $\Gamma\left(S D_{8 n}\right)$ is not hyperenergetic when odd $n \geq 15$ or even $n \geq 2$.

Firstly, we can obtain the adjacency matrix of $\Gamma\left(S D_{8 n}\right)$ in the following. When $n$ is odd, then $\Gamma\left(S D_{8 n}\right)=K_{4} \vee\left(n K_{4} \cup K_{4 n-4}\right)$, to derive the adjacency matrix of a commuting graph $\Gamma\left(S D_{8 n}\right)$, we first put the elements of $Z\left(S D_{8 n}\right)$, then $\Phi_{3}$ and finally $\Phi_{2}$ respectively, and get the following matrix is

$$
A\left(\Gamma\left(S D_{8 n}\right)\right)=\left[\begin{array}{cc}
A\left(\Gamma\left(C_{4 n}\right)\right) & H_{4 n} \\
H_{4 n}^{T} & F_{4 n}
\end{array}\right]
$$

where

$$
A\left(\Gamma\left(C_{4 n}\right)\right)=\left[\begin{array}{ccccc}
0 & 1 & 1 & \cdots & 1 \\
1 & 0 & 1 & \cdots & 1 \\
1 & 1 & 0 & \cdots & 1 \\
\vdots & \vdots & \vdots & & \vdots \\
1 & 1 & 1 & \cdots & 0
\end{array}\right], \quad H_{4 n}=\left[\begin{array}{cccccc}
1 & 1 & 1 & 1 & \cdots & 1 \\
1 & 1 & 1 & 1 & \cdots & 1 \\
1 & 1 & 1 & 1 & \cdots & 1 \\
1 & 1 & 1 & 1 & \cdots & 1 \\
\vdots & \vdots & \vdots & \vdots & & \vdots \\
0 & 0 & 0 & 0 & \cdots & 0
\end{array}\right]
$$

and

$$
E=\left[\begin{array}{llll}
0 & 1 & 1 & 1 \\
1 & 0 & 1 & 1 \\
1 & 1 & 0 & 1 \\
1 & 1 & 1 & 0
\end{array}\right], \quad F_{4 n}=\left[\begin{array}{llll}
E & & & \\
& E & & \\
& & \ddots & \\
& & & E
\end{array}\right],
$$

where $A\left(\Gamma\left(C_{4 n}\right)\right), H_{4 n}$ and $F_{4 n}$ are of order $4 n$.

When $n$ is even, then we have $\Gamma\left(S D_{8 n}\right)=K_{2} \vee\left(2 n K_{2} \cup K_{4 n-2}\right)$. Thus

$$
A\left(\Gamma\left(S D_{8 n}\right)\right)=\left[\begin{array}{cc}
A\left(\Gamma\left(C_{4 n}\right)\right) & \Upsilon_{4 n} \\
Y_{4 n}^{T} & Z_{4 n}
\end{array}\right]
$$

where

$$
Y_{4 n}=\left[\begin{array}{cccc}
1 & 1 & \cdots & 1 \\
1 & 1 & \cdots & 1 \\
\vdots & \vdots & \ddots & \vdots \\
0 & 0 & \cdots & 0
\end{array}\right], \quad Z_{4 n}=\left[\begin{array}{cc}
0 & I_{2 n} \\
I_{2 n} & 0
\end{array}\right] .
$$

Here, $A\left(\Gamma\left(C_{4 n}\right)\right), Y_{4 n}$ and $Z_{4 n}$ are all of order $4 n$.

Theorem 1. For odd $n \geq 3$, the characteristic polynomial of $\Gamma\left(S D_{8 n}\right)$ is

$$
p(\lambda)=(\lambda-3)^{n-1}(\lambda+1)^{7 n-2}[(\lambda-4 n+1)(\lambda-3)(\lambda+1)-16 n(\lambda-4 n+5)] .
$$

Proof. By Theorem 2.2 of [10], we can get

$$
\begin{aligned}
p(\lambda) & =\left|\lambda I_{8 n}-A\left(\Gamma\left(S D_{8 n}\right)\right)\right| \\
& =\left|\begin{array}{cc}
\lambda I_{4 n}-A\left(\Gamma\left(C_{4 n}\right)\right) & -H_{4 n} \\
-H_{4 n}^{T} & \lambda I_{4 n}-F_{4 n}
\end{array}\right| \\
& =\left|\lambda I_{4 n}-F_{4 n}\right| \cdot\left|\lambda I_{4 n}-A\left(\Gamma\left(C_{4 n}\right)\right)-H_{4 n}\left(\lambda I_{4 n}-F_{4 n}\right)^{-1} H_{4 n}^{T}\right| .
\end{aligned}
$$


Setting $e=(1,1,1,1)^{T}$. By directed computation, Theorem 2.5 of [10] gives

$$
\left(\lambda I_{4}-E\right)^{-1}=\left((\lambda+1) I_{4}-e e^{T}\right)^{-1}=\left[\begin{array}{cccc}
a & b & b & b \\
b & a & b & b \\
b & b & a & b \\
b & b & b & a
\end{array}\right],
$$

where $a=\frac{2-\lambda}{(\lambda+1)(3-\lambda)}$ and $b=-\frac{1}{(\lambda+1)(3-\lambda)}$. Therefore,

$$
\begin{aligned}
& \lambda I_{4 n}-A\left(\Gamma\left(C_{4 n}\right)\right)-H_{4 n}\left(\lambda I_{4 n}-F\right)^{-1} H_{4 n}^{T} \\
& {\left[\begin{array}{ccccccc}
\lambda+\frac{4 n}{3-\lambda} & -1+\frac{4 n}{3-\lambda} & -1+\frac{4 n}{3-\lambda} & -1+\frac{4 n}{3-\lambda} & -1 & \cdots & -1 \\
-1+\frac{4 n}{3-\lambda} & \lambda+\frac{4 n}{3-\lambda} & -1+\frac{4 n}{3-\lambda} & -1+\frac{4 n}{3-\lambda} & -1 & \cdots & -1 \\
-1+\frac{4 n}{3-\lambda} & -1+\frac{4 n}{3-\lambda} & \lambda+\frac{4 n}{3-\lambda} & -1+\frac{4 n}{3-\lambda} & -1 & \cdots & -1 \\
-1+\frac{4 n}{3-\lambda} & -1+\frac{4 n}{3-\lambda} & -1+\frac{4 n}{3-\lambda} & \lambda+\frac{4 n}{3-\lambda} & -1 & \cdots & -1 \\
-1 & -1 & -1 & -1 & \lambda & \cdots & -1 \\
\vdots & \vdots & \vdots & \vdots & \vdots & & \vdots \\
-1 & -1 & -1 & -1 & -1 & \cdots & \lambda
\end{array}\right] .}
\end{aligned}
$$

Applying a series of row and column operations, that is, for $i=2,3,4$, subtracting the $i$-th row from the $(i-1)$-th row, and subtracting the $i$-th column from the $(i-1)$-th column yields to

$$
\begin{aligned}
& \left|\lambda I_{4 n}-A\left(\Gamma\left(C_{4 n}\right)\right)-H_{4 n}\left(\lambda I_{4 n}-F\right)^{-1} H_{4 n}^{T}\right| \\
& =\left|\begin{array}{ccccccc}
2(\lambda+1) & -1-\lambda & 0 & 0 & 0 & \cdots & 0 \\
-1-\lambda & 2(\lambda+1) & -1-\lambda & 0 & 0 & \cdots & 0 \\
0 & -1-\lambda & 2(\lambda+1) & -1-\lambda & 0 & \cdots & 0 \\
0 & 0 & -1-\lambda & \lambda+\frac{4 n}{3-\lambda} & -1 & \cdots & -1 \\
0 & 0 & 0 & -1 & \lambda & \cdots & -1 \\
\vdots & \vdots & \vdots & \vdots & \vdots & & \vdots \\
0 & 0 & 0 & -1 & -1 & \cdots & \lambda
\end{array}\right| \\
& =2(\lambda+1) \Lambda_{1}-(1+\lambda)^{2} \Lambda_{2},
\end{aligned}
$$

where

$$
\Lambda_{1}=\left|\begin{array}{cccccc}
2(\lambda+1) & -1-\lambda & 0 & 0 & \cdots & 0 \\
-1-\lambda & 2(\lambda+1) & -1-\lambda & 0 & \cdots & 0 \\
0 & -1-\lambda & \lambda+\frac{4 n}{3-\lambda} & -1 & \cdots & -1 \\
0 & 0 & -1 & \lambda & \cdots & -1 \\
\vdots & \vdots & \vdots & \vdots & & \vdots \\
0 & 0 & -1 & -1 & \cdots & \lambda
\end{array}\right|_{4 n-1}
$$

and

$$
\Lambda_{2}=\left|\begin{array}{ccccc}
2(\lambda+1) & -1-\lambda & 0 & \cdots & 0 \\
-1-\lambda & \lambda+\frac{4 n}{3-\lambda} & -1 & \cdots & -1 \\
0 & -1 & \lambda & \cdots & -1 \\
\vdots & \vdots & \vdots & & \vdots \\
0 & -1 & -1 & \cdots & \lambda
\end{array}\right|_{4 n-2} .
$$

For notational convenience, we denote

$$
\Lambda_{3}=\left|\begin{array}{cccc}
\lambda+\frac{4 n}{3-\lambda} & -1 & \cdots & -1 \\
-1 & \lambda & \cdots & -1 \\
\vdots & \vdots & & \vdots \\
-1 & -1 & \cdots & \lambda
\end{array}\right|_{4 n-3} .
$$


Next, we are going to compute the values of $\Lambda_{1}, \Lambda_{2}$ and $\Lambda_{3}$. Since

$$
\begin{aligned}
\Lambda_{3} & =\left|\begin{array}{cccc}
\lambda & -1 & \cdots & -1 \\
-1 & \lambda & \cdots & -1 \\
\vdots & \vdots & & \vdots \\
-1 & -1 & \cdots & \lambda
\end{array}\right|_{4 n-3}+\left|\begin{array}{cccc}
\frac{4 n}{3-\lambda} & -1 & \cdots & -1 \\
0 & \lambda & \cdots & -1 \\
\vdots & \vdots & & \vdots \\
0 & -1 & \cdots & \lambda
\end{array}\right|_{4 n-3} \\
& =(\lambda-4 n+4)(\lambda+1)^{4 n-4}+\frac{4 n}{3-\lambda}(\lambda-4 n+5)(\lambda+1)^{4 n-5} .
\end{aligned}
$$

Therefore

$$
\begin{aligned}
\Lambda_{2} & =2(\lambda+1) \Lambda_{3}-(1+\lambda)^{2} \cdot(\lambda-4 n+5)(\lambda+1)^{4 n-5}, \\
& =(\lambda-4 n+3)(\lambda+1)^{4 n-3}+\frac{8 n}{3-\lambda}(\lambda-4 n+5)(\lambda+1)^{4 n-4} .
\end{aligned}
$$

And then

$$
\begin{aligned}
\Lambda_{1} & =2(\lambda+1) \Lambda_{2}-(1+\lambda)^{2} \Lambda_{3} \\
& =(\lambda-4 n+2)(\lambda+1)^{4 n-2}+\frac{12 n}{3-\lambda}(\lambda-4 n+5)(\lambda+1)^{4 n-3} .
\end{aligned}
$$

After calculations, we can get

$$
\begin{aligned}
& \left|\lambda I_{4 n}-A\left(\Gamma\left(C_{4 n}\right)\right)-H_{4 n}\left(\lambda I_{4 n}-F_{4 n}\right)^{-1} H_{4 n}^{T}\right| \\
& =2(\lambda+1) \Lambda_{1}-(1+\lambda)^{2} \Lambda_{2} \\
& =(\lambda-4 n+1)(\lambda+1)^{4 n-1}+\frac{16 n}{3-\lambda}(\lambda-4 n+5)(\lambda+1)^{4 n-2},
\end{aligned}
$$

which together with $\left|\lambda I_{4 n}-F_{4 n}\right|=(\lambda-3)^{n}(\lambda+1)^{3 n}$ yields the desired result.

Corollary 1. For odd $n \geq 15$, the energy of $\Gamma\left(S D_{8 n}\right)$ is given below,

$$
14 n-4<E\left(\Gamma\left(S D_{8 n}\right)\right)<16 n-4 .
$$

Consequently, the commuting graph $\Gamma\left(S D_{8 n}\right)$ with odd $n \geq 15$ is not hyperenergetic.

Proof. Consider the cubic equation

$$
(\lambda-4 n+1)(\lambda-3)(\lambda+1)-16 n(\lambda-4 n+5)]=0,
$$

i.e.,

$$
\lambda^{3}-(4 n+1) \lambda^{2}-(8 n+5) \lambda+\left(64 n^{2}-68 n-3\right)=0 .
$$

Then, by Theorem 1 , the equation has three real roots $\lambda_{1}, \lambda_{2}, \lambda_{3}$. And we can assume that $\lambda_{1} \geq \lambda_{2} \geq \lambda_{3}$. Then, for odd $n \geq 15$, we have

$$
\lambda_{1}+\lambda_{2}+\lambda_{3}=4 n+1>0
$$

and

$$
\lambda_{1} \cdot \lambda_{2} \cdot \lambda_{3}=-\left(64 n^{2}-68 n-3\right)<0 .
$$

Therefore, by (1) and (2), we have $\lambda_{1}<0$ and $\lambda_{2}, \lambda_{3}>0$.

Let

$$
f(\lambda)=\lambda^{3}-(4 n+1) \lambda^{2}-(8 n+5) \lambda+\left(64 n^{2}-68 n-3\right) .
$$


Then we have

$$
\begin{gathered}
f(0)=64 n^{2}-68 n-3>0, \\
f(-n)=-5 n^{3}+71 n^{2}-63 n-3=(71-5 n) n^{2}-63 n-3<0,
\end{gathered}
$$

for odd $n \geq 15$. And $f(\lambda)$ is continuous on $[-n, 0]$. Therefore, by the intermediate value theorem, we have

$$
-n<\lambda_{1}<0
$$

Hence, by (1) and (3), we get

$$
4 n+1<\left|\lambda_{1}\right|+\left|\lambda_{2}\right|+\left|\lambda_{3}\right|=\left(\lambda_{1}+\lambda_{2}+\lambda_{3}\right)-2 \lambda_{1}<(4 n+1)+2 n=6 n+1 .
$$

And, by Theorem 1, we have

$$
E\left(\Gamma\left(S D_{8 n}\right)\right)=3(n-1)+(7 n-2)+\left|\lambda_{1}\right|+\left|\lambda_{2}\right|+\left|\lambda_{3}\right|=10 n-5+\left|\lambda_{1}\right|+\left|\lambda_{2}\right|+\left|\lambda_{3}\right| .
$$

So, for odd $n \geq 15$, we get

$$
14 n-4=(10 n-5)+(4 n+1)<E\left(\Gamma\left(S D_{8 n}\right)\right)<(10 n-5)+(6 n+1)=16 n-4 .
$$

Since $E\left(\Gamma\left(S D_{8 n}\right)\right)<16 n-4<2(8 n-1)$, we have the commuting graph $\Gamma\left(S D_{8 n}\right)$ with odd $n \geq 15$ is not hyperenergetic.

Corollary 2. For odd $n \geq 3$, the spectral radius of $\Gamma\left(S D_{8 n}\right)$ satisfies the following,

$$
\lambda_{1}\left(\Gamma\left(C_{4 n}\right)\right)<\lambda_{1}\left(\Gamma\left(S D_{8 n}\right)\right) \leq \lambda_{1}\left(\Gamma\left(C_{4 n}\right)\right)+4 \sqrt{2 n}
$$

Proof. It is clear from Proposition 1.3.9 of [11] that $\lambda_{1}\left(\Gamma\left(C_{4 n}\right)\right)<\lambda_{1}\left(\Gamma\left(S D_{8 n}\right)\right)$. Let us divide the adjacency matrix into two parts, i.e. $A\left(\Gamma\left(S D_{8 n}\right)\right)=\mathcal{T}+\mathcal{W}$, where $\mathcal{T}, \mathcal{W}$ are symmetric matrices of the form

$$
\mathcal{T}=\left[\begin{array}{cc}
A\left(\Gamma\left(C_{4 n}\right)\right) & 0_{4 n} \\
0_{4 n} & F_{4 n}
\end{array}\right] \text { and } \mathcal{W}=\left[\begin{array}{cc}
0_{4 n} & H_{4 n} \\
H_{4 n}^{T} & 0_{4 n}
\end{array}\right]
$$

By Theorem 1.3.15 of [11], we have

$$
\lambda_{1}\left(\Gamma\left(S D_{8 n}\right)\right) \leq \lambda_{1}(\mathcal{T})+\lambda_{1}(\mathcal{W})=\lambda_{1}\left(\Gamma\left(C_{4 n}\right)\right)+\lambda_{1}(\mathcal{W})
$$

It is clear that $\mathcal{W}$ is the adjacency matrix of $K_{4,4 n}$ together with $4 n-4$ number of isolated nodes. Therefore, $\lambda_{1}(\mathcal{W})=4 \sqrt{2 n}$. Hence, we get

$$
\lambda_{1}\left(\Gamma\left(C_{4 n}\right)\right)<\lambda_{1}\left(\Gamma\left(S D_{8 n}\right)\right) \leq \lambda_{1}\left(\Gamma\left(C_{4 n}\right)\right)+4 \sqrt{2 n}
$$

This completes the proof.

Theorem 2. For even $n \geq 2$, the characteristic polynomial of $\Gamma\left(S D_{8 n}\right)$ is

$$
p(\lambda)=(\lambda-1)^{2 n-1}(\lambda+1)^{6 n-2}\left[\left(\lambda^{2}-8 n-1\right)(\lambda-4 n+3)-2\left(\lambda^{2}-1\right)\right] .
$$

Proof. By Theorem 2.2 of [10], we get

$$
\begin{aligned}
p(\lambda) & =\left|\begin{array}{cc}
\lambda I_{4 n}-A\left(\Gamma\left(C_{4 n}\right)\right) & -Y_{4 n} \\
-Y_{4 n}^{T} & \lambda I_{4 n}-Z_{4 n}
\end{array}\right| \\
& =\left|\lambda I_{4 n}-Z_{4 n}\right| \cdot\left|\lambda I_{4 n}-A\left(\Gamma\left(C_{4 n}\right)\right)-Y_{4 n}\left(\lambda I_{4 n}-Z_{4 n}\right)^{-1} Y_{4 n}^{T}\right| .
\end{aligned}
$$


After some computations, we have $\left|\lambda I_{4 n}-Z_{4 n}\right|=\left(\lambda^{2}-1\right)^{2 n}$, and the Theorem 2.4 of [10] gives

$$
\left(\lambda I_{4 n}-Z_{4 n}\right)^{-1}=\left[\begin{array}{ll}
\lambda I_{2 n} & -I_{2 n} \\
-I_{2 n} & \lambda I_{2 n}
\end{array}\right]^{-1}=\left[\begin{array}{ll}
\frac{\lambda}{\lambda^{2}-1} I_{2 n} & \frac{1}{\lambda^{2}-1} I_{2 n} \\
\frac{1}{\lambda^{2}-1} I_{2 n} & \frac{\lambda}{\lambda^{2}-1} I_{2 n}
\end{array}\right]
$$

Then

$$
Y_{4 n}\left(\lambda I_{4 n}-Z_{4 n}\right)^{-1} Y_{4 n}^{T}=\left[\begin{array}{ccccc}
\frac{4 n}{\lambda-1} & \frac{4 n}{\lambda-1} & 0 & \cdots & 0 \\
\frac{4 n}{\lambda-1} & \frac{4 n}{\lambda-1} & 0 & \cdots & 0 \\
0 & 0 & 0 & \cdots & 0 \\
\vdots & \vdots & \vdots & \ddots & \vdots \\
0 & 0 & 0 & \cdots & 0
\end{array}\right]
$$

Thus, we get

$$
\begin{aligned}
& \left|\lambda I_{4 n}-A\left(\Gamma\left(C_{4 n}\right)\right)-Y_{4 n}\left(\lambda I_{4 n}-Z_{4 n}\right)^{-1} Y_{4 n}^{T}\right| \\
& =\left|\begin{array}{ccccc}
\lambda-\frac{4 n}{\lambda-1} & -1-\frac{4 n}{\lambda-1} & -1 & \cdots & -1 \\
-1-\frac{4 n}{\lambda-1} & \lambda-\frac{4 n}{\lambda-1} & -1 & \cdots & -1 \\
-1 & -1 & \lambda & \cdots & -1 \\
\vdots & \vdots & \vdots & \ddots & \vdots \\
-1 & -1 & -1 & \cdots & \lambda
\end{array}\right|
\end{aligned}
$$

By subtracting the second row from the first row and subtracting the second column from the first column, we get

$$
\begin{aligned}
& \left|\lambda I_{4 n}-A\left(\Gamma\left(C_{4 n}\right)\right)-\Upsilon_{4 n}\left(\lambda I_{4 n}-Z_{4 n}\right)^{-1} \Upsilon_{4 n}^{T}\right| \\
& =\left|\begin{array}{ccccc}
2(\lambda+1) & -1-\lambda & 0 & \cdots & 0 \\
-1-\lambda & \lambda-\frac{4 n}{\lambda-1} & -1 & \cdots & -1 \\
0 & -1 & \lambda & \cdots & -1 \\
\vdots & \vdots & \vdots & & \vdots \\
0 & -1 & -1 & \cdots & \lambda
\end{array}\right| \\
& =2(\lambda+1) \Omega_{1}-(1+\lambda)^{2} \Omega_{2},
\end{aligned}
$$

where

$$
\Omega_{1}=\left|\begin{array}{cccc}
\lambda-\frac{4 n}{\lambda-1} & -1 & \cdots & -1 \\
-1 & \lambda & \cdots & -1 \\
\vdots & \vdots & \ddots & \vdots \\
-1 & -1 & \cdots & \lambda
\end{array}\right|_{4 n-1} \quad, \quad \Omega_{2}=\left|\begin{array}{cccc}
\lambda & -1 & \cdots & -1 \\
-1 & \lambda & \cdots & -1 \\
\vdots & \vdots & \ddots & \vdots \\
-1 & -1 & \cdots & \lambda
\end{array}\right|_{4 n-2}
$$

Directed calculation gives

$$
\begin{aligned}
\Omega_{1} & =\left|\begin{array}{cccc}
\lambda & -1 & \cdots & -1 \\
-1 & \lambda & \cdots & -1 \\
\vdots & \vdots & \ddots & \vdots \\
-1 & -1 & \cdots & \lambda
\end{array}\right|_{4 n-1}+\left|\begin{array}{cccc}
-\frac{4 n}{\lambda-1} & -1 & \cdots & -1 \\
0 & \lambda & \cdots & -1 \\
\vdots & \vdots & \ddots & \vdots \\
0 & -1 & \cdots & \lambda
\end{array}\right|_{4 n-1} \\
& =(\lambda-4 n+2)(\lambda+1)^{4 n-2}-\frac{4 n}{\lambda-1}(\lambda-4 n+3)(\lambda+1)^{4 n-3} .
\end{aligned}
$$

and

$$
\Omega_{2}=(\lambda-4 n+3)(\lambda+1)^{4 n-3} .
$$


We now conclude that

$$
\begin{aligned}
& \left|\lambda I_{4 n}-A\left(\Gamma\left(C_{4 n}\right)\right)-Y_{4 n}\left(\lambda I_{4 n}-Z_{4 n}\right)^{-1} Y_{4 n}^{T}\right| \\
& =2(\lambda+1) \Omega_{1}-(1+\lambda)^{2} \Omega_{2} \\
& =(\lambda-1)^{-1}(\lambda+1)^{4 n-2}\left[\left(\lambda^{2}-8 n-1\right)(\lambda-4 n+3)-2\left(\lambda^{2}-1\right)\right] .
\end{aligned}
$$

Hence, the required result immediately follows.

Corollary 3. For even $n \geq 2$, the energy of $\Gamma\left(S D_{8 n}\right)$ satisfies the following,

$$
12 n-4<E\left(\Gamma\left(S D_{8 n}\right)\right)<16 n-4 .
$$

Consequently, the commuting graph $\Gamma\left(S D_{8 n}\right)$ with even $n \geq 2$ is not hyperenergetic.

Proof. Consider the cubic equation

$$
\left.\left(\lambda^{2}-8 n-1\right)(\lambda-4 n+3)-2\left(\lambda^{2}-1\right)\right]=0,
$$

i.e.,

$$
\lambda^{3}-(4 n-1) \lambda^{2}-(8 n+1) \lambda+\left(32 n^{2}-20 n-1\right)=0 .
$$

Then, by Theorem 2 , the equation has three real roots $\lambda_{1}, \lambda_{2}, \lambda_{3}$. And we can assume that $\lambda_{1} \geq \lambda_{2} \geq \lambda_{3}$. Then, for even $n \geq 2$, we have

$$
\lambda_{1}+\lambda_{2}+\lambda_{3}=4 n-1>0
$$

and

$$
\lambda_{1} \cdot \lambda_{2} \cdot \lambda_{3}=-\left(32 n^{2}-20 n-1\right)<0 .
$$

Therefore, by (4) and (5), we have $\lambda_{1}<0$ and $\lambda_{2}, \lambda_{3}>0$.

Let

$$
f(\lambda)=\lambda^{3}-(4 n-1) \lambda^{2}-(8 n+1) \lambda+\left(32 n^{2}-20 n-1\right) .
$$

Then we have

$$
\begin{gathered}
f(0)=32 n^{2}-20 n-1>0, \\
f(-2 n)=-24 n^{3}+52 n^{2}-18 n-1=(52-24 n) n^{2}-18 n-1<0,
\end{gathered}
$$

for even $n \geq 2$. Furthermore, $f(\lambda)$ is continuous on $[-2 n, 0]$. Therefore, by the intermediate value theorem, we have

$$
-2 n<\lambda_{1}<0
$$

Hence, by (4) and (6), we get

$$
4 n-1<\left|\lambda_{1}\right|+\left|\lambda_{2}\right|+\left|\lambda_{3}\right|=\left(\lambda_{1}+\lambda_{2}+\lambda_{3}\right)-2 \lambda_{1}<(4 n-1)+4 n=8 n-1 .
$$

In addition, by Theorem 2, we have

$$
E\left(\Gamma\left(S D_{8 n}\right)\right)=(2 n-1)+(6 n-2)+\left|\lambda_{1}\right|+\left|\lambda_{2}\right|+\left|\lambda_{3}\right|=8 n-3+\left|\lambda_{1}\right|+\left|\lambda_{2}\right|+\left|\lambda_{3}\right| .
$$

So, for even $n \geq 2$, we get

$$
12 n-4=(8 n-3)+(4 n-1)<E\left(\Gamma\left(S D_{8 n}\right)\right)<(8 n-3)+(8 n-1)=16 n-4 .
$$

Since $E\left(\Gamma\left(S D_{8 n}\right)\right)<16 n-4<2(8 n-1)$, we have the commuting graph $\Gamma\left(S D_{8 n}\right)$ with even $n \geq 2$ is not hyperenergetic. 
Corollary 4 . For even $n \geq 2$, the spectral radius of $\Gamma\left(S D_{8 n}\right)$ satisfies the following,

$$
\lambda_{1}\left(\Gamma\left(C_{4 n}\right)\right)<\lambda_{1}\left(\Gamma\left(S D_{8 n}\right)\right) \leq \lambda_{1}\left(\Gamma\left(C_{4 n}\right)\right)+2 \sqrt{2 n}
$$

Proof. Similar to the proof of Theorem 2.

\section{Laplacian Spectral Properties of Commuting Graphs}

In this section, we are going to find the Laplacian spectrum of commuting graphs on semidihedral groups, and then we compute the Laplacian energy and the number of spanning trees.

Firstly, we obtain the Laplacian matrix of $\Gamma\left(S D_{8 n}\right)$ when $n$ is odd. Now we have $\Gamma\left(S D_{8 n}\right)=K_{4} \vee\left(n K_{4} \cup K_{4 n-4}\right)$. For notational convenience, we define

$$
B=\left[\begin{array}{ccccc}
0 & 1 & 1 & \cdots & 1 \\
1 & 0 & 1 & \cdots & 1 \\
1 & 1 & 0 & \cdots & 1 \\
\vdots & \vdots & \vdots & & \vdots \\
1 & 1 & 1 & \cdots & 0
\end{array}\right], \quad C=\left[\begin{array}{cccccc}
1 & 1 & 1 & 1 & \cdots & 1 \\
1 & 1 & 1 & 1 & \cdots & 1 \\
1 & 1 & 1 & 1 & \cdots & 1 \\
1 & 1 & 1 & 1 & \cdots & 1 \\
\vdots & \vdots & \vdots & \vdots & & \vdots \\
0 & 0 & 0 & 0 & \cdots & 0
\end{array}\right]
$$

and

$$
E=\left[\begin{array}{llll}
0 & 1 & 1 & 1 \\
1 & 0 & 1 & 1 \\
1 & 1 & 0 & 1 \\
1 & 1 & 1 & 0
\end{array}\right], \quad F=\left[\begin{array}{llll}
E & & & \\
& E & & \\
& & \ddots & \\
& & & E
\end{array}\right]
$$

where $B, C$ and $F$ are of order $4 n$. And then we denote

$$
K=\left[\begin{array}{ccccccc}
8 n-1 & -1 & -1 & -1 & -1 & \ldots & -1 \\
-1 & 8 n-1 & -1 & -1 & -1 & \ldots & -1 \\
-1 & -1 & 8 n-1 & -1 & -1 & \ldots & -1 \\
-1 & -1 & -1 & 8 n-1 & -1 & \ldots & -1 \\
-1 & -1 & -1 & -1 & 4 n-1 & \ldots & -1 \\
\vdots & \vdots & \vdots & \vdots & \vdots & & \vdots \\
-1 & -1 & -1 & -1 & -1 & \ldots & 4 n-1
\end{array}\right],
$$

then the Laplacian matrix of $\Gamma\left(S D_{8 n}\right)$ is given as

$$
L=\left[\begin{array}{cc}
K & -C \\
-C^{T} & 7 I_{4 n}-F
\end{array}\right]
$$

Theorem 3. For odd $n \geq 2$, the characteristic polynomial of the Laplacian matrix of $\Gamma\left(S D_{8 n}\right)$ is

$$
p_{L}(\lambda)=\lambda(\lambda-4)^{n}(\lambda-8)^{3 n}(\lambda-8 n)^{4}(\lambda-4 n)^{4 n-5} .
$$

Proof. By Theorem 2.2 of [10], we get

$$
\begin{aligned}
p_{L}(\lambda) & =\left|\lambda I_{8 n}-L\right|=\left|\begin{array}{cc}
\lambda I_{4 n}-K & C \\
C^{T} & \lambda I_{4 n}-7 I_{4 n}+F
\end{array}\right| \\
& =\left|(\lambda-7) I_{4 n}+F\right| \cdot\left|\lambda I_{4 n}-K-C\left((\lambda-7) I_{4 n}+F\right)^{-1} C^{T}\right| .
\end{aligned}
$$


Upon calculation, we get from Theorem 2.5 of [10] that

$$
\left((\lambda-7) I_{4}+E\right)^{-1}=\left((\lambda-8) I_{4}+e e^{T}\right)^{-1}=\left[\begin{array}{llll}
d & c & c & c \\
c & d & c & c \\
c & c & d & c \\
c & c & c & d
\end{array}\right],
$$

where $c=-\frac{1}{(\lambda-8)(\lambda-4)}$ and $d=\frac{\lambda-5}{(\lambda-8)(\lambda-4)}$. Hence,

$$
\begin{aligned}
& \lambda I_{4 n}-K-C\left((\lambda-7) I_{4 n}+F\right)^{-1} C^{T} \\
& =\left[\begin{array}{ccccccc}
\lambda-8 n+1-\frac{4 n}{\lambda-4} & 1-\frac{4 n}{\lambda-4} & 1-\frac{4 n}{\lambda-4} & 1-\frac{4 n}{\lambda-4} & 1 & \cdots & 1 \\
1-\frac{4 n}{\lambda-4} & \lambda-8 n+1-\frac{4 n}{\lambda-4} & 1-\frac{4 n}{\lambda-4} & 1-\frac{4 n}{\lambda-4} & 1 & \cdots & 1 \\
1-\frac{4 n}{\lambda-4} & 1-\frac{4 n}{\lambda-4} & \lambda-8 n+1-\frac{4 n}{\lambda-4} & 1-\frac{4 n}{\lambda-4} & 1 & \cdots & 1 \\
1-\frac{4 n}{\lambda-4} & 1-\frac{4 n}{\lambda-4} & 1-\frac{4 n}{\lambda-4} & \lambda-8 n+1-\frac{4 n}{\lambda-4} & 1 & \cdots & 1 \\
1 & 1 & 1 & 1 & \lambda-4 n+1 & \ldots & 1 \\
\vdots & \vdots & \vdots & \vdots & \vdots & & \vdots \\
1 & 1 & 1 & 1 & 1 & \cdots & \lambda-4 n+1
\end{array}\right] .
\end{aligned}
$$

Applying a series of row and column operations, that is, for $i=2,3,4$, subtracting the $i$-th row from the $(i-1)$-th row, and subtracting the $i$-th column from the $(i-1)$-th column. Then

$$
\begin{aligned}
& \left|\lambda I_{4 n}-K-C\left((\lambda-7) I_{4 n}+F\right)^{-1} C^{T}\right| \\
& =\left|\begin{array}{ccccccc}
2(\lambda-8 n) & -\lambda+8 n & 0 & 0 & 0 & \cdots & 0 \\
-\lambda+8 n & 2(\lambda-8 n) & -\lambda+8 n & 0 & 0 & \cdots & 0 \\
0 & -\lambda+8 n & 2(\lambda-8 n) & -\lambda+8 n & 0 & \cdots & 0 \\
0 & 0 & -\lambda+8 n & \lambda-8 n+1-\frac{4 n}{\lambda-4} & 1 & \cdots & 1 \\
0 & 0 & 0 & 1 & \lambda-4 n+1 & \cdots & 1 \\
\vdots & \vdots & \vdots & \vdots & \vdots & & \vdots \\
0 & 0 & 0 & 1 & 1 & \cdots & \lambda-4 n+1
\end{array}\right| \\
& =2(\lambda-8 n) \Delta_{1}-(-\lambda+8 n)^{2} \Delta_{2},
\end{aligned}
$$

where

$$
\Delta_{1}=\left|\begin{array}{cccccc}
2(\lambda-8 n) & -\lambda+8 n & 0 & 0 & \cdots & 0 \\
-\lambda+8 n & 2(\lambda-8 n) & -\lambda+8 n & 0 & \cdots & 0 \\
0 & -\lambda+8 n & \lambda-8 n+1-\frac{4 n}{\lambda-4} & 1 & \cdots & 1 \\
0 & 0 & 1 & \lambda-4 n+1 & \cdots & 1 \\
\vdots & \vdots & \vdots & \vdots & & \vdots \\
0 & 0 & 1 & 1 & \cdots & \lambda-4 n+1
\end{array}\right|_{4 n-1}
$$

and

$$
\Delta_{2}=\left|\begin{array}{ccccc}
2(\lambda-8 n) & -\lambda+8 n & 0 & \cdots & 0 \\
-\lambda+8 n & \lambda-8 n+1-\frac{4 n}{\lambda-4} & 1 & \cdots & 1 \\
0 & 1 & \lambda-4 n+1 & \cdots & 1 \\
\vdots & \vdots & \vdots & & \vdots \\
0 & 1 & 1 & \cdots & \lambda-4 n+1
\end{array}\right|_{4 n-2}
$$

For notational convenience, we set

$$
\Delta_{3}=\left|\begin{array}{cccc}
\lambda-8 n+1-\frac{4 n}{\lambda-4} & 1 & \cdots & 1 \\
1 & \lambda-4 n+1 & \cdots & 1 \\
\vdots & \vdots & & \vdots \\
1 & 1 & \cdots & \lambda-4 n+1
\end{array}\right|_{4 n-3} .
$$


We now are ready to compute the values of $\Delta_{1}, \Delta_{2}$ and $\Delta_{3}$. Note that

$$
\begin{aligned}
\Delta_{3} & =\left|\begin{array}{cccc}
\lambda-4 n+1 & 1 & \cdots & 1 \\
1 & \lambda-4 n+1 & \cdots & 1 \\
\vdots & \vdots & & \vdots \\
1 & 1 & \cdots & \lambda-4 n+1
\end{array}\right|_{4 n-3}\left|\begin{array}{cccc}
-4 n-\frac{4 n}{\lambda-4} & 1 & \cdots & 1 \\
0 & \lambda-4 n+1 & \cdots & 1 \\
\vdots & \vdots & & \vdots \\
0 & 1 & \cdots & \lambda-4 n+1
\end{array}\right|_{4 n-3} \\
& =(\lambda-3)(\lambda-4 n)^{4 n-4}-4 n(\lambda-3)(\lambda-4 n)^{4 n-5} .
\end{aligned}
$$

Thus,

$$
\begin{aligned}
\Delta_{2} & =2(\lambda-8 n) \Delta_{3}-(-\lambda+8 n)^{2} \cdot(\lambda-4)(\lambda-4 n)^{4 n-5} \\
& =2(\lambda-8 n)(\lambda-3)(\lambda-4 n)^{4 n-4}-\left(\lambda^{2}-4 \lambda+8 n\right)(\lambda-8 n)(\lambda-4 n)^{4 n-5} .
\end{aligned}
$$

And then we have

$$
\begin{aligned}
\Delta_{1} & =2(\lambda-8 n) \Delta_{2}-(-\lambda+8 n)^{2} \Delta_{3} \\
& =3(\lambda-3)(\lambda-8 n)^{2}(\lambda-4 n)^{4 n-4}-2\left(\lambda^{2}-(2 n+4) \lambda+14 n\right)(\lambda-8 n)^{2}(\lambda-4 n)^{4 n-5} .
\end{aligned}
$$

Combining $\Delta_{1}$ and $\Delta_{2}$, it follows that

$$
\begin{aligned}
& \left|\lambda I_{4 n}-K-C\left((\lambda-7) I_{4 n}+F\right)^{-1} C^{T}\right| \\
& =2(\lambda-8 n) \Delta_{1}-(\lambda-8 n)^{2} \Delta_{2} \\
& =\lambda(\lambda-8 n)^{4}(\lambda-4 n)^{4 n-5},
\end{aligned}
$$

which together with $\left|(\lambda-7) I_{4 n}+F\right|=(\lambda-4)^{n}(\lambda-8)^{3 n}$ leads to the required result.

As byproducts of Theorem 3, we obtain the following corollaries.

Corollary 5. For odd $n \geq 2$, the Laplacian spectrum of $\Gamma\left(S D_{8 n}\right)$ is

$$
\text { L-spec }\left(\Gamma\left(S D_{8 n}\right)\right)=\left\{0^{1}, 4^{n}, 8^{3 n},(4 n)^{4 n-5},(8 n)^{4}\right\} .
$$

Corollary 6. For odd $n \geq 2$, the Laplacian energy of $\Gamma\left(S D_{8 n}\right)$ is

$$
L E\left(\Gamma\left(S D_{8 n}\right)\right)=16 n^{2}+24 n-32 .
$$

Corollary 7. For odd $n \geq 2$, the number of spanning trees of $\Gamma\left(S D_{8 n}\right)$ is

$$
\operatorname{st}\left(\Gamma\left(S D_{8 n}\right)\right)=2^{19 n-1} n^{4 n-2} \text {. }
$$

Proof. The proof is straightforward by using Corollary 5 and Proposition 1.3.4 of [12] .

Finally, we are ready to compute the Laplacian spectrum of commuting graphs of $S D_{8 n}$ when $n$ is even, then we have $\Gamma\left(S D_{8 n}\right)=K_{2} \vee\left(2 n K_{2} \cup K_{4 n-2}\right)$. For notational convenience, we first define

$$
X=\left[\begin{array}{cccc}
0 & 1 & \cdots & 1 \\
1 & 0 & \cdots & 1 \\
\vdots & \vdots & \ddots & \vdots \\
1 & 1 & \cdots & 0
\end{array}\right], \quad Y=\left[\begin{array}{cccc}
1 & 1 & \cdots & 1 \\
1 & 1 & \cdots & 1 \\
\vdots & \vdots & \ddots & \vdots \\
0 & 0 & \cdots & 0
\end{array}\right], \quad Z=\left[\begin{array}{cc}
0 & I_{2 n} \\
I_{2 n} & 0
\end{array}\right]
$$


Here, $X, Y$ and $Z$ are all of order $4 n$. We then denote $V=3 I_{4 n}-Z$ and

$$
U=\left[\begin{array}{ccccc}
8 n-1 & -1 & -1 & \cdots & -1 \\
-1 & 8 n-1 & -1 & \cdots & -1 \\
-1 & -1 & 4 n-1 & \cdots & -1 \\
\vdots & \vdots & \vdots & & \vdots \\
-1 & -1 & -1 & \cdots & 4 n-1
\end{array}\right]
$$

then

$$
L=\left[\begin{array}{cc}
U & -Y \\
-Y^{T} & V
\end{array}\right]
$$

Theorem 4. For even $n \geq 2$, the characteristic polynomial of the Laplacian matrix of $\Gamma\left(S D_{8 n}\right)$ is

$$
p_{L}(\lambda)=\lambda(\lambda-4)^{2 n}(\lambda-2)^{2 n}(\lambda-4 n)^{4 n-3}(\lambda-8 n)^{2} .
$$

Proof. By Theorem 2.2 of [10], we have

$$
\begin{aligned}
p_{L}(\lambda) & =\left|\lambda I_{8 n}-L\right|=\left|\begin{array}{cc}
\lambda I_{4 n}-U & Y \\
Y^{T} & \lambda I_{4 n}-V
\end{array}\right| \\
& =\left|\lambda I_{4 n}-V\right| \cdot\left|\lambda I_{4 n}-U-Y\left(\lambda I_{4 n}-V\right)^{-1} Y^{T}\right| .
\end{aligned}
$$

Upon computations, we get $\left|\lambda I_{4 n}-V\right|=\left((\lambda-3)^{2}-1\right)^{2 n}$, and Theorem 2.4 of [10] yields

$$
\left(\lambda I_{4 n}-V\right)^{-1}=\left[\begin{array}{cc}
(\lambda-3) I_{2 n} & I_{2 n} \\
I_{2 n} & (\lambda-3) I_{2 n}
\end{array}\right]^{-1}=\left[\begin{array}{ll}
\frac{\lambda-3}{(\lambda-3)^{2}-1} I_{2 n} & \frac{-1}{(\lambda-3)^{2}-1} I_{2 n} \\
\frac{-1}{(\lambda-3)^{2}-1} I_{2 n} & \frac{\lambda-3}{(\lambda-3)^{2}-1} I_{2 n}
\end{array}\right] .
$$

Then

$$
\begin{aligned}
& \left|\lambda I_{4 n}-U-Y\left(\lambda I_{4 n}-V\right)^{-1} Y^{T}\right| \\
& =\left|\begin{array}{ccccc}
\lambda-8 n+1-\frac{4 n}{\lambda-2} & 1-\frac{4 n}{\lambda-2} & 1 & \cdots & 1 \\
1-\frac{4 n}{\lambda-2} & \lambda-8 n+1-\frac{4 n}{\lambda-2} & 1 & \cdots & 1 \\
1 & 1 & \lambda-4 n+1 & \cdots & 1 \\
\vdots & \vdots & \vdots & \ddots & \vdots \\
1 & 1 & 1 & \cdots & \lambda-4 n+1
\end{array}\right| \\
& =\left|\begin{array}{ccccc}
2(\lambda-8 n) & -\lambda+8 n & 0 & \cdots & 0 \\
-\lambda+8 n & \lambda-8 n+1-\frac{4 n}{\lambda-2} & 1 & \cdots & 1 \\
0 & 1 & \lambda-4 n+1 & \cdots & 1 \\
\vdots & \vdots & \vdots & \ddots & \vdots \\
0 & 1 & 1 & \cdots & \lambda-4 n+1
\end{array}\right| \\
& =2(\lambda-8 n) \Psi_{1}-(8 n-\lambda)^{2} \Psi_{2},
\end{aligned}
$$

where

$$
\Psi_{1}=\left|\begin{array}{cccc}
\lambda-8 n+1-\frac{4 n}{\lambda-2} & 1 & \cdots & 1 \\
1 & \lambda-4 n+1 & \cdots & 1 \\
\vdots & \vdots & \ddots & \vdots \\
1 & 1 & \cdots & \lambda-4 n+1
\end{array}\right|_{4 n-1}
$$

and

$$
\Psi_{2}=\left|\begin{array}{cccc}
\lambda-4 n+1 & 1 & \cdots & 1 \\
1 & \lambda-4 n+1 & \cdots & 1 \\
\vdots & \vdots & \ddots & \vdots \\
1 & 1 & \cdots & \lambda-4 n+1
\end{array}\right|_{4 n-2}
$$


It is not hard to find that

$$
\Psi_{1}=(\lambda-1)(\lambda-4 n)^{4 n-2}-\left(4 n+\frac{4 n}{\lambda-2}\right)(\lambda-2)(\lambda-4 n)^{4 n-3},
$$

and

$$
\Psi_{2}=(\lambda-2)(\lambda-4 n)^{4 n-3} .
$$

Therefore, we conclude that

$$
\begin{aligned}
& \left|\lambda I_{4 n}-U-Y\left(\lambda I_{4 n}-V\right)^{-1} Y^{T}\right| \\
& =2(\lambda-8 n) \Psi_{1}-(8 n-\lambda)^{2} \Psi_{2} \\
& =\lambda(\lambda-8 n)^{2}(\lambda-4 n)^{4 n-3} .
\end{aligned}
$$

This completes the proof.

From Theorem 4, one could get the following corollaries similarly.

Corollary 8. For even $n \geq 2$, the Laplacian spectrum of $\Gamma\left(S D_{8 n}\right)$ is

$$
\text { L-spec }\left(\Gamma\left(S D_{8 n}\right)\right)=\left\{0^{1}, 4^{2 n}, 2^{2 n},(4 n)^{4 n-3},(8 n)^{2}\right\} .
$$

Corollary 9. For even $n \geq 2$, the Laplacian energy of $\Gamma\left(S D_{8 n}\right)$ is

$$
\operatorname{LE}\left(\Gamma\left(S D_{8 n}\right)\right)=16 n^{2}-4 n+4 .
$$

Corollary 10. For even $n \geq 2$, the number of spanning trees of $\Gamma\left(S D_{8 n}\right)$ is

$$
\operatorname{st}\left(\Gamma\left(S D_{8 n}\right)\right)=2^{14 n-3} n^{4 n-2} .
$$

\section{Connectivity and Planarity of Commuting Graphs}

In this section, we show that these commuting graphs are not Hamiltonian. Furthermore, we discuss vertex connectivity, the planarity, and minimum disconnecting set of commuting graphs of semidihedral groups.

Theorem 5. Suppose $S D_{8 n}$ is a semidihedral group and $\Gamma\left(S D_{8 n}\right)$ is the commuting graph on $S D_{8 n}$, for $n \geq 2$. Then

1. For odd $n, \kappa\left(\Gamma\left(S D_{8 n}\right)\right)=4$;

2. For even $n, \kappa\left(\Gamma\left(S D_{8 n}\right)\right)=2$;

3. $\Gamma\left(S D_{8 n}\right)$ is not Hamiltonian for both odd and even $n$.

Proof. (1) For an odd integer $n$, since we know that the center $Z\left(S D_{8 n}\right)=\left\{e, a^{n}, a^{2 n}, a^{3 n}\right\}$ of $S D_{8 n}$ is adjacent to all other vertices of $\Gamma\left(S D_{8 n}\right)$, so using Remark 1 , we have the graph becomes disconnected into $n+1$ components by deletion of $Z\left(S D_{8 n}\right)$. Therefore, $\kappa\left(\Gamma\left(S D_{8 n}\right)\right)=4$.

(2) For an even integer $n$, since we know that the center $Z\left(S D_{8 n}\right)=\left\{e, a^{2 n}\right\}$ of $S D_{8 n}$ is adjacent to all other vertices of $\Gamma\left(S D_{8 n}\right)$, so using Remark 1 , the graph becomes disconnected into $2 n+1$ components by deletion of $Z\left(S D_{8 n}\right)$. Therefore, $\kappa\left(\Gamma\left(S D_{8 n}\right)\right)=2$.

(3) For an odd integer $n \geq 2$, we have $\left|Z\left(S D_{8 n}\right)\right|=4$ and the components of $\Gamma\left(S D_{8 n}\right)-$ $Z\left(S D_{8 n}\right)$ are $n+1 \geq 4$. Also, for an even integer $n$, we have $\left|Z\left(S D_{8 n}\right)\right|=2$ and the components of $\Gamma\left(S D_{8 n}\right)-Z\left(S D_{8 n}\right)$ are $2 n+1 \geq 2$. Therefore, by Proposition 7.2.3 of [13], we have $\Gamma\left(S D_{8 n}\right)$ is not Hamiltonian.

Theorem 6. Let $\Gamma\left(S D_{8 n}\right)$ be the commuting graph of the semidihedral group $S D_{8 n}$ for $n \geq 2$. Then $\Gamma\left(S D_{8 n}\right)$ is nonplanar. 
Proof. Note that $\langle a\rangle$ is cyclic subgroup of order $4 n$. It suffices to show that the commuting graph $\Gamma\left(S D_{8 n}\right)$ is nonplanar if and only if the subgraph induced by $\langle a\rangle$ is nonplanar. Since $\langle a\rangle$ is cyclic, $\Gamma\left(S D_{8 n}\right)$ is nonplanar for $n \geq 2$.

Theorem 7. For odd $n \geq 5$, we have $\sigma\left(\Gamma\left(S D_{8 n}\right)\right)=7$. Moreover, for every $0 \leq i \leq$ $n-1$, we have $\mathbb{E}\left[b a^{i},\left\{e, a^{n}, a^{2 n}, a^{3 n}, b a^{n+i}, b a^{2 n+i}, b a^{3 n+i}\right\}\right], \mathbb{E}\left[b a^{n+i},\left\{e, a^{n}, a^{2 n}, a^{3 n}, b a^{i}, b a^{2 n+i}\right.\right.$, $\left.\left.b a^{3 n+i}\right\}\right], \mathbb{E}\left[b a^{2 n+i},\left\{e, a^{n}, a^{2 n}, a^{3 n}, b a^{i}, b a^{n+i}, b a^{3 n+i}\right\}\right]$, and $\mathbb{E}\left[b a^{3 n+i},\left\{e, a^{n}, a^{2 n}, a^{3 n}, b a^{i}, b a^{n+i}\right.\right.$, $\left.\left.b a^{2 n+i}\right\}\right]$ are minimum disconnecting sets of $\Gamma\left(S D_{8 n}\right)$.

Proof. Suppose $u \in \Phi_{1}$, so $d(u) \geq \phi(4 n)+1 \geq 9$. Now let $v \in \Phi_{2}$, the only vertices adjacent to $v$ in $\Gamma\left(S D_{8 n}\right)$ are elements of $\Phi_{2} \backslash\{v\}$, and so $d(v)=7$. Hence, we conclude that $\sigma\left(\Gamma\left(S D_{8 n}\right)\right)=7$.

It is clear from the structure of $\Gamma\left(S D_{8 n}\right)$ when $n$ is odd, $\mathbb{N}\left(b a^{i}\right)=\left\{e, a^{n}, a^{2 n}, a^{3 n}, b a^{n+i}\right.$, $\left.b a^{2 n+i}, b a^{3 n+i},\right\}$ where $0 \leq i \leq n-1$. Since $\sigma\left(\Gamma\left(S D_{8 n}\right)\right)=d\left(b a^{i}\right)=7$, we have $\mathbb{E}\left[b a^{i},\left\{e, a^{n}\right.\right.$, $\left.\left.a^{2 n}, a^{3 n}, b a^{n+i}, b a^{2 n+i}, b a^{3 n+i}\right\}\right]$ is the minimum disconnecting set of $\Gamma\left(S D_{8 n}\right)$. Similarly, we have $\mathbb{E}\left[b a^{n+i},\left\{e, a^{n}, a^{2 n}, a^{3 n}, b a^{i}, b a^{2 n+i}, b a^{3 n+i}\right\}\right], \mathbb{E}\left[b a^{2 n+i},\left\{e, a^{n}, a^{2 n}, a^{3 n}, b a^{i}, b a^{n+i}\right.\right.$, $\left.\left.b a^{3 n+i}\right\}\right]$, and $\mathbb{E}\left[b a^{3 n+i},\left\{e, a^{n}, a^{2 n}, a^{3 n}, b a^{i}, b a^{n+i}, b a^{2 n+i}\right\}\right]$ are minimum disconnecting sets of $\Gamma\left(S D_{8 n}\right)$.

Theorem 8. For even $n \geq 2$, we have $\sigma\left(\Gamma\left(S D_{8 n}\right)\right)=3$. Moreover, for every $0 \leq i \leq 2 n-1$, we have $\mathbb{E}\left[b a^{i},\left\{e, a^{2 n}, a^{2 n+i}\right\}\right]$ and $\mathbb{E}\left[b a^{2 n+i},\left\{e, a^{2 n}, b a^{i}\right\}\right]$ are minimum disconnecting sets of $\Gamma\left(S D_{8 n}\right)$.

Proof. Similar to the proof of Theorem 7.

Author Contributions: Conceptualization, T.C., W.L., F.E.-S., Y.L. and M.D.; investigation, W.L. and M.D.; data analysis, F.E.-S. and T.C.; writing the paper, M.D.; Y.L.; supervision, T.C., Y.L. and M.D.; project administration, M.D. All authors have read and agreed to the published version of the manuscript.

Funding: Weijun Liu was supported by NSFC (Grant Nos. 11871479, 12071484), Hunan Provincial Natural Science Foundation (2020JJ4675, 2018JJ2479) and Mathematics and Interdisciplinary Sciences Project of CSU. Matthias Dehmer thanks the Austrian Science Foundadtion to support this work (P 30031).

Institutional Review Board Statement: Not applicable.

Informed Consent Statement: Not applicable.

Conflicts of Interest: The authors declare no conflict of interest.

Sample Availability: Samples of the compounds are available from the authors.

\section{References}

1. Bundy, D. The connectivity of commuting Graphs. J. Combin. Theory Ser. A 2006, 113, 995-1007. [CrossRef]

2. Ali, F.; Li, Y. The connectivity and the spectral radius of commuting graphs on certain finite groups. Linear Multilinear Algebra, 2019. [CrossRef]

3. Cheng, T.; Feng, L.; Huang, H. Integral Cayley graphs over dicyclic group. Linear Algebra Appl. 2019, 566, 121-137. [CrossRef]

4. Cheng, T.; Feng, L.; Liu, W. Directed Strongly Regular Cayley Graphs over Metacyclic Groups of Order 4n. Mathematics 2019, 7, 1011. [CrossRef]

5. Gutman, I. The energy of a graph. Ber. Math. Stat. Sekt. Forschungszent. Graz. 1978, 103, 1-22.

6. Li, X.; Shi, Y.; Gutman, I. Graph Energy; Springer: New York, NY, USA, 2012.

7. Walikar, H.B.; Gutman, I.; Hampiholi, P.R.; Ramane, H.S. Non-hyperenergetic graphs. Graph Theory Notes N. Y. 2001, XLI, 14-16.

8. Mohar, B. The Laplacian spectrum of graphs. In Graph Theory, Combinatorics and Applications, 2nd ed.; Alavi, Y., Chartrand, G., Oellermann, O.R., Schwenk, A.J., Eds.; Wiley: Hoboken, NJ, USA, 1991, 871-898.

9. Harary, F. Graph Theory; Addision-Wesley Reading: Boston, MA, USA, 1969.

10. Zhang, F. Matrix Theory: Basic Results and Techniques, 2nd ed; Springer: New York, NY, USA, 2011.

11. Cvetković, D.; Rowlinson, P.; Simić, S. An Introduction to the Theory of Graph Spectra; Cambridge University Prese: London, UK, 2010. 
12. Brouwer, A.E.; Haemers, W.H. Spectra of Graphs; Springer: New York, NY, USA, 2012.

13. West, D.B. Introduction to Graph Theory; Prentice-Hall: New Delhi, India, 2003. 